\section{Leipziger Allerlei 2019}



Liebe Kolleginnen, liebe Kollegen,

... bitte einatmen, ausatmen und nicht mehr atmen... - eine von uns MTRAs häufig benutzte Anweisung an den Patienten, mit der sicher der ein oder andere schon mal im Privaten von Außenstehenden angesprochen wurde. Dieses Atemkommando ist aber für uns enorm wichtig, um qualitativ gute und bewegungsarme Bilder zu erzeugen, vor allem beim Thorax.

Welche typischen Diagnosen und Befunde findet man denn in der Lunge, in der von uns am häufigsten erstellten Röntgenaufnahme? Wichtig genug, um dafür eine ganze Sitzung inklusive der Schnittbilddiagnostik zu organisieren, aber auch, wenn man mal über den radiologischen Tellerrand hinausschaut ins Fachgebiet der Bronchoskopie.
Das $A$ und $O$ einer MTRA sind Kenntnisse über eine gute Einstelltechnik. Dass diese nicht immer einfach zu realisieren ist, vor allem bei traumatisierten Patienten, ist klar. Trotzdem sollten eventuelle Fehlermöglichkeiten bekannt sein. Dieses Mal beschäftigen wir uns zu diesem Thema mit den oberen Extremitäten, also von der Schulter bis zum Handgelenk.

Auch die Neuroradiologie hat reichlich an Themen zu bieten. Was ist der Unterschied zwischen Morbus Alzheimer und der Demenz? Ist die Myelografie eigentlich noch up-to-date oder hat die Schnittbilddiagnostik in diesem Fachgebiet mittlerweile das Zepter übernommen? Wann ist es denn sinnvoll, welches Untersuchungsverfahren zu wählen? Was passiert an einem kleinen, nicht neuroradiologischen Schwerpunkt-
Krankenhaus bei einem neuroradiologischen Notfall? Auch wieder eine interessante Sitzung, die sicherlich für jeden thematisch etwas bereithält.

Auch der letzte Programmpunkt: „....alles Tele oder was?" ist ein aktueller Blick in die Zukunft. Wird eigentlich alles immer weiter digitalisiert und über das Internet ablaufen? Welche Möglichkeiten und Chancen verspricht die Arztsprechstunde im Internet, aber auch welche Risiken? Welche Neuigkeiten gibt es bei der Nutzung der Teleradiologie?

Das 9. Leipziger Allerlei ist also wieder ein bunter Mix aus Themen und Vorträgen.

Wir hoffen, damit Ihr Interesse geweckt zu haben und Sie am 26. Januar 2019 in Leipzig begrüßen zu dürfen.

Ihre Claudia Mundry und Birgit Lenz 